\title{
Solution of the Grazing Goat Problem: A Conflict between Beauty and Pragmatism
}

\author{
Robert J Marks II \\ DOI: 10.33014 /issn.2640-5652.3.2.marks.1
}

\section{Abstract}

What is the ideal solution of a problem in mathematics? It depends on your nerd ideology. Pure mathematicians worship the beauty of a mathematics result. Closed form solutions are particularly beautiful. Engineers and applied mathematicians, on the other hand, focus on the result independent of its beauty. If a solution exists and can be calculated, that's enough. The job is done. An example is solution of the grazing goat problem. A recent closed form solution in the form of a ratio of two contour integrals has been found for the grazing goat problem and its beauty has been admired by pure mathematicians. For the engineer and applied mathematician, numerical solution of the grazing goat problem comes from an easily derived transcendental equation. The transcendental equation, known for some time, was not considered a beautiful enough solution for the pure mathematician so they kept on looking until they found a closed form solution. The numerical evaluation of the transcendental equation is not as beautiful. It is not in closed form. But the accuracy of the solution can straightforwardly be evaluated to within any accuracy desired. To illustrate, we derive and solve the transcendental equation for a generalization of the grazing goat problem.

The grazing goat problem is a simply posed exercise in geometry that has been floating around for over two and a half centuries since at least 1748. Nadis gives a concise history of the problem (Nadis, 2020). A circular fence encloses a field of grass. A goat is tied to fence. How long is the leash such that the goat has access to one half of the grass within the circle?

Mathematicians have searched for an elegant closed form solution to the problem. We will show the problem can be solved straightforwardly to any accuracy using the solution to a transcendental equation. The answer is that the tether length is 1.15872847301812 times that of the circle's radius. The answer is neither beautiful nor exact. The true tether length, probably irrational, goes on forever and, like $\pi$ or $\sqrt{2}$, can be computed to any accuracy desired. If the radius of the circular fence is ten meters, the number 1.15872847301812 provides

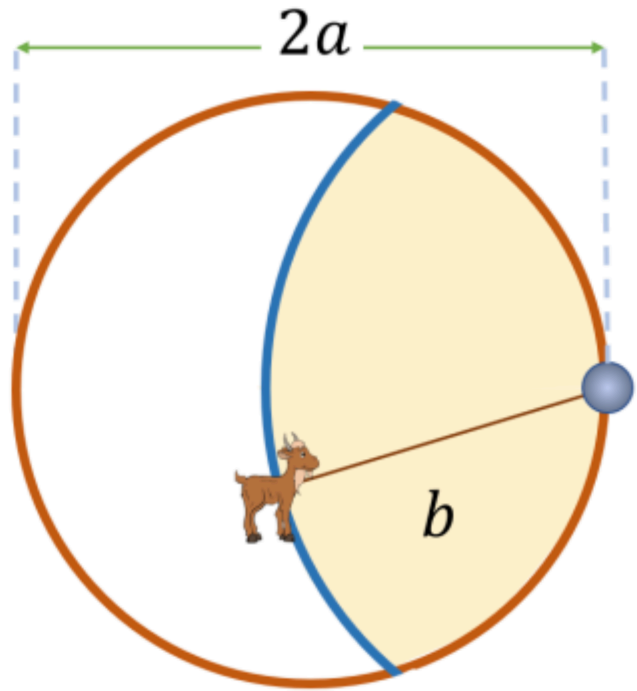

A fence is circular with radius $a$. How long, $b$, should the tether be so the goat has access to one half of the area inside the fence?

Figure 1: The Grazing Goat Problem

accuracy of the tether length to less than a millionth of a millionth of a meter. Isn't this good enough? It depends on your nerd ideology.

\section{Background}

Mathematicians have been looking for a closed form solution for the grazing goat problem albeit unsuccessfully. Why a closed form solution? Because it is beautiful. The geometric infinite sum $\sum_{n=0}^{\infty} x^{n}$ has a beautiful closed form solution of $(1-x)^{-1}$ as long as $|x|<1$. Mathematicians celebrate such beauty.

Paul Erdős celebrated the most beautiful of mathematical proofs as being in "God's book" (Andreescu and Dospinescu, 2010). Proving whether or not a proof is in "God's book" is not a mathematical problem itself but is, rather, a subjective 
call.

Likewise, the definition of a closed form mathematical solution is fuzzy. There are attempts to define closed form (Borwein and Crandall, 2013) but there is no widespread agreement on a precise mathy definition. Everyone agrees that the solution of the geometric series is in closed form. But what about the integral of a Gaussian curve, like $\int_{0}^{y} e^{-x^{2}} d x$ ? When there is no convenient solution, a new transcendental function is often defined. In case of the Gaussian integral, the solution is defined something like $\operatorname{erf}(y)$ where the erf stands for "error function." The error function is available in most computer languages and even in spread sheets. In the eyes of a mathematician, does this make the Gaussian integral solution in terms of erf a closed form solution?

Here's a similar example closer to home. In a paper currently under review, we required evaluation of the infinite series

$$
\varphi(z)=\sum_{k=0}^{\infty} z^{k} \log _{2} k !
$$

We could find no closed form simplification but did show the series converged for $|z|<1$. The value of $\varphi(z)$ can then be evaluated to any accuracy desired, just like the number $\pi$. By defining $\varphi(z)$, can we say the series has a closed form solution like we do using $\operatorname{erf}(y)$ ?

Even more fundamentally, is it meaningful to ask for a closed form solution for a constant like $\pi$ ? Restricting closed form operations to predefined rules, Chow 1999 defines a closed form solution of $\pi$ by

$$
\pi=\sqrt{-1} \log (-1)
$$

Going to such lengths to quantify closed form seems, to this author, silly. Like Erdös's idea of "God's book," the idea of closed form is best kept as a subjective judgement of beauty.

There are obvious examples in the extreme. All agree that most puppies are cute. On the other side, there is a "World's Ugliest Dog Contest." Most dogs lie between the extremes of cute and ugly.

Similarly in math, when there is an answer, all agree that closed form solutions to many Diophantine equations don't exist. Solutions to Diophantine equations are ugly. On the other extreme, we also agree that Euler's solution of the Basel problem is in a beautiful, closed form. Solving a problem that had eluded mathematicians for decades, Euler proved that

$$
\sum_{n=1}^{\infty} \frac{1}{n^{2}}=\frac{\pi^{2}}{6}
$$

This is unquestionably an elegant closed form solution that deserves to be appreciated for its beauty.

Here's a more personal more difficult example. I was a coauthor on a paper presenting the first and thus far only closed form solution describing the performance of a Neyman-Pearson optimal detector in the presence of non-Gaussian noise (Marks Il et al., 1978). The result, I thought, was beautiful. But over a decade later, C.W. Helstrom noted

\begin{abstract}
"Marks, Wise, Haldeman and Whited derived a closed-form expression for the complementary cumulative probability distribution ... Their formula involves a triple summation, the number of terms of which increase with $n$ like $n^{3}$, and the terms alternate in sign." (Helstrom, 1989, emphasis added)
\end{abstract}

When trying to calculate our beautiful formula on a computer, Helstrom noted the requirement of using the differences between very large numbers which can be problematic. For example, computing the number 2 using the difference between

1010003137838752886587533208381420617177669147303598253490428755468731152 and

1010003137838752886587533208381420617177669147303598253490428755468731150

is troubling because high computational accuracy required. The differenced numbers in our closed form solution became larger and larger for higher order solutions.

Helstrom solved the same problem using digital evaluation of a contour integral. The solution was no longer in closed form, but there were no requirements of subtraction of large numbers that differed only at the least significant digits.

So which solution of the optimal detection problem was best? It depends on your nerd ideology. To the pure mathematician, my closed form solution was best. Evaluation required a finite number of terms defined using well know components and familiar math operations. Given unbounded computational resources, the answer is exact. To the engineer and applied mathematician, Helstrom's answer is best. Lower computational resources can be applied to compute a result to high computational accuracy.

Which brings us back to the grazing goat problem. A recent paper claimed to derive a closed form solution to the goat grazing problem (Ullisch, 2020). But the solution was in the form 


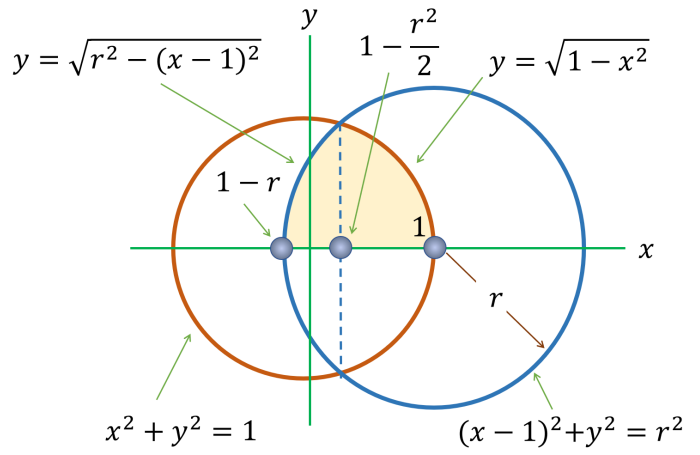

Figure 2: The geometry of the grazing goat problem

of the ratio of two contour integrals which had to be evaluated numerically. Some of the beauty of the closed form solution of the goat grazing problem is lost when such digital evaluation is required. As we now show, a computationally accessible solution of the grazing goat problem has been available for a long time.

\section{Grazing Goat Problem Solution}

The simple geometry of the grazing goat problem is shown in Figure 2. The goat fence is inside a circle with unit radius. The unknown goat tether is assumed to be $r$ units long. There is no loss of generality in assuming the fenced circle has unit radius. If the fence radius is $a$, then the desired length of the tether is $r a$.

The circle of the fence has the familiar equation $x^{2}+y^{2}=1$ and the circle swept by the goat tether is $(x-1)^{2}+y^{2}=r^{2}$. Subtracting these two equations and solving for $x$ gives the intersection point $1-\frac{r^{2}}{2}$ as shown in Figure 1 . Also shown in Figure 2 is the functional form of the two circle equations, namely $y=\sqrt{1-x^{2}}$ and $y=\sqrt{r^{2}-(x-1)^{2}}$. The problem is now a straightforward integration problem. The desired shaded area under the curve, shown shaded in Figure 2, is one half of the grazing area available to the goat, namely $\frac{\pi}{4}$. From Figure 2, the problem is the solution to

$$
\rho(r)=\int_{1-r}^{1-\frac{r^{2}}{2}} \sqrt{r^{2}-(x-1)^{2}} d x+\int_{1-\frac{r^{2}}{2}}^{1} \sqrt{1-x^{2}} d x .
$$

where we would like to find the value of $r$ so that $\rho(r)=\frac{\pi}{4}$.

The two indefinite integrals over the shaded area in Figure 2 can be evaluated as

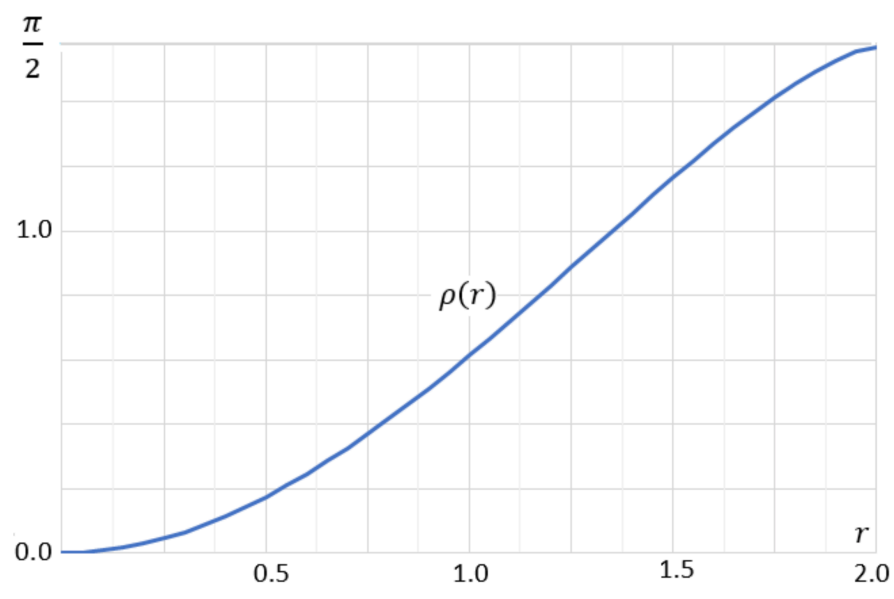

Figure 3: The plot of $\rho(r)$ in Equation 2

$$
\int^{x} \sqrt{1-x^{2}} d x=\frac{1}{2} \sqrt{1-x^{2}} x+\frac{1}{2} \arcsin (x)
$$

and

$$
\begin{aligned}
& \int^{x} \sqrt{r^{2}-(x-1)^{2}} d x \\
& =\frac{1}{2}(x-1) \sqrt{r^{2}-(x-1)^{2}}+\frac{1}{2} r^{2} \arcsin \left(\frac{x-1}{r}\right) .
\end{aligned}
$$

The equation in (1) then, as described in Ullisch (2020), becomes

$$
\begin{aligned}
\rho(r) & =\left.\frac{1}{2}\left[(x-1) \sqrt{r^{2}-(x-1)^{2}}+r^{2} \arcsin \left(\frac{x-1}{r}\right)\right]\right|_{x=1-r} ^{1-\frac{r^{2}}{2}} \\
& +\left.\frac{1}{2}\left[\sqrt{1-x^{2}} x+\frac{1}{2} \arcsin (x)\right]\right|_{x=1-\frac{r^{2}}{2}} ^{1}
\end{aligned}
$$

We could make the substitutions for the lower and upper limits shown in (2) but find it easier to let the computer do it.

A plot of $\rho(r)$ is shown in Figure 3. The function is strictly increasing. This is also evident from Figure 2 where the shaded area clearly increases as $r$ increases reaching a maximum value of 2 giving a half grazing area of $\frac{\pi}{2}$.

There are many techniques to solve the for $r$ in (2) when $\rho(r)=\frac{\pi}{4}$. Newton's method, taught in introductory calculus, comes to mind. A computationally simpler approach, not needing the derivative required by Newton's method, is interval halving also known as binary search. 
The idea is simple as is seen in the straightforward algorithm here:

1. Set low $=0$

2. Set high $=2$

3. Evaluate the midpoint $r=\frac{\text { high+low }}{2}$

4. If error $=\rho(r)-\frac{\pi}{4}>0$, set high $=r$

5. Otherwise, set low $=r$

6. Go to Step 3

The iteration continues until the desired accuracy, or when the error, constrained to the computational accuracy of the computer, is zero.

Looking at Figure 3, an eyeball estimate of $\rho(r)=\frac{\pi}{4} \approx 0.79$ safely places $r$ between low $=1.00$ and high $=1.25$ so the interval halving algorithm could be initiated there. But the interval halving algorithm converges quickly and broader initialization only require a few more iterations. Every iteration reduces the interval on which the solution by half. Initially, the solution is known to be on the interval $(0,2)$. The second iteration reduces the interval to $(1,2)$. Then to $(1,1.5),(1,1.25)$ and $(1.125,1.25)$. The length of these intervals respectively are $\left[2,1, \frac{1}{2}, \frac{1}{4} \cdot \frac{1}{8}\right]$. If $I=2$ is the width of the initial interval, the width of the interval after the $N$ th iteration is

$$
\text { Accuracy }=I \times 2^{-N}
$$

The algorithm zooms in on the correct answer quickly.

The iteration for the grazing goat problem converges to $r=1.15872847301812$ after 49 iterations to zero error when the algorithm is performed with the accuracy of a MS Excel spread sheet. The solution uses 14 digits to the right of the decimal consistent with (3) where Accuracy $=2 \times 2^{-49} \approx$ $10^{-15}$. The result is the same with the digitally calculated "closed form" contour integration solution given by Ullisch but boasts two more digits of accuracy. The last few digits of accuracy can be rightfully questioned, however, because of the accuracy of Excel at this precision.

\section{Grazing Goat Problem Inversion}

The grazing goat problem results can be easily generalized to the case where the goat is allowed to eat $p \%$ of the available grazing area inside of the circular fence. The solution thus

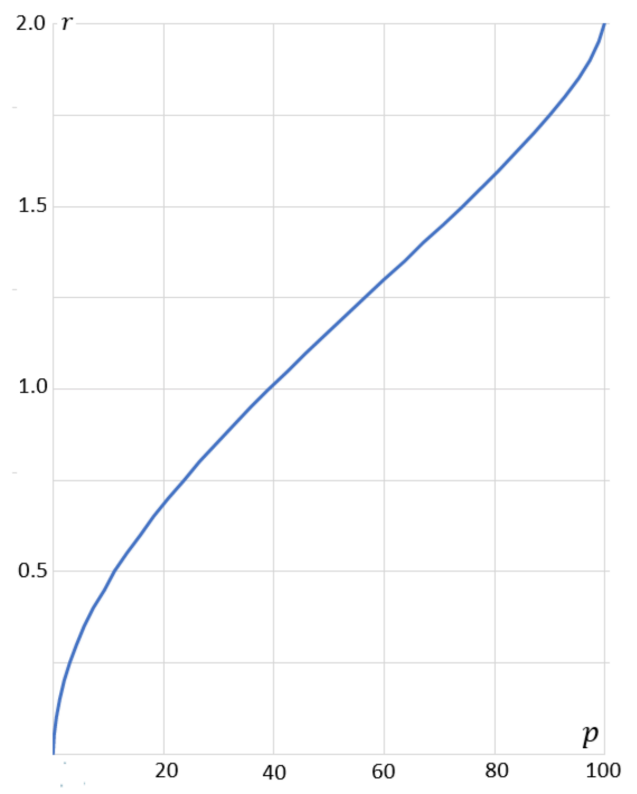

Instead of the grazing area, the percent $p$ of half the available grazing area in the circular fence is used. $p=100 \%$ corresponds to an area of $\frac{\pi}{2}$.

Figure 4: Inversion of the $\rho(r)$ in Figure 3

far has concentrated on the specific case of $p=50 \%$ of the grazing area. In equation (2), the problem to solve is now

$$
\rho(r)=\frac{p}{100} \frac{\pi}{2}
$$

The original problem is for $p=50 \%$ so that $\rho(r)=\frac{\pi}{4}$.

The radius $r$ as a function of $p$ is shown in Figure 4. All strictly increasing functions have a unique inverse, e.g. $y=e^{x}$ and $x=\log y$. The plot in Figure 4 is the inverse of Figure 3 except, instead of the true area, the percent of area $p$ is used.

\section{Final Thought}

Nineteenth century romantic poet John Keats said, "Beauty is truth and truth beauty." In terms of the solution of mathematical problems, truth in a solution may not be beautiful. Closed form solutions are beautiful but in the case of accurate solutions to mathematical problems, like the grazing goat problem, truth need not be beautiful.

\section{Communications Blyth Institute}




\section{References}

Andreescu, Titu and Gabriel Dospinescu (2010). Problems from the Book. 2nd Edition. XYZ Press.

Borwein, Jonathan M and Richard E Crandall (2013). "Closed Forms: What They are and Why We Care". In: Notices of the AMS 60.1, pp. 50-65. URL: https://citeseerx . ist. psu . edu/viewdoc/download?doi=10.1.1.190. $4817 \% 5$ C\&rep $=$ rep $1 \% 5$ C\&type $=$ pdf.

Chow, Timothy Y (1999). "What is a Closed-Form Number?" In: The American Mathematical Monthly 106.5, pp. 440448. URL: https://www.tandfonline.com/doi/pdf/ $10.1080 / 00029890.1999 .12005066$ ? casa_token $=$ V9Kvgbh4 exMAAAAA : cVLDnRUzoUR9iW14739rU3bm _ KZj7n5PPoNbfHTgJWSevq9poaHMSs7oJiN TtLJS54TxxgiotMO.

Helstrom, C W (1989). "Detectability of Signals in Laplace Noise". In: IEEE Transactions on Aerospace and Electronic Systems AES-25.2, pp. 190-196.

Marks II, R J et al. (1978). "Detection in Laplace Noise". In: IEEE Transactions on Aerospace and Electronic Systems AES-14, pp. 866-872. DOI: 10.1109/TAES.1978. 308550. URL: https : / / ieeexplore . ieee . org / document/4102075.

Nadis, Steve (2020). "After Centuries, a Seemingly Simple Math Problem Gets an Exact Solution". In: Quanta Magazine. URL: https : / / www . quantamagazine . org / mathematician-solves - centuries-old-grazinggoat-problem-exactly-20201209/.

Ullisch, Ingo (2020). "A Closed-Form Solution to the Geometric Goat Problem". In: Mathematical Intelligencer 42, pp. 1-5. DOI: $10.1007 / \mathrm{s} 00283-020-09966-0$. URL: https : / / link . springer . com/article/ $10.1007 \%$ 2Fs00283-020-09966-0. 